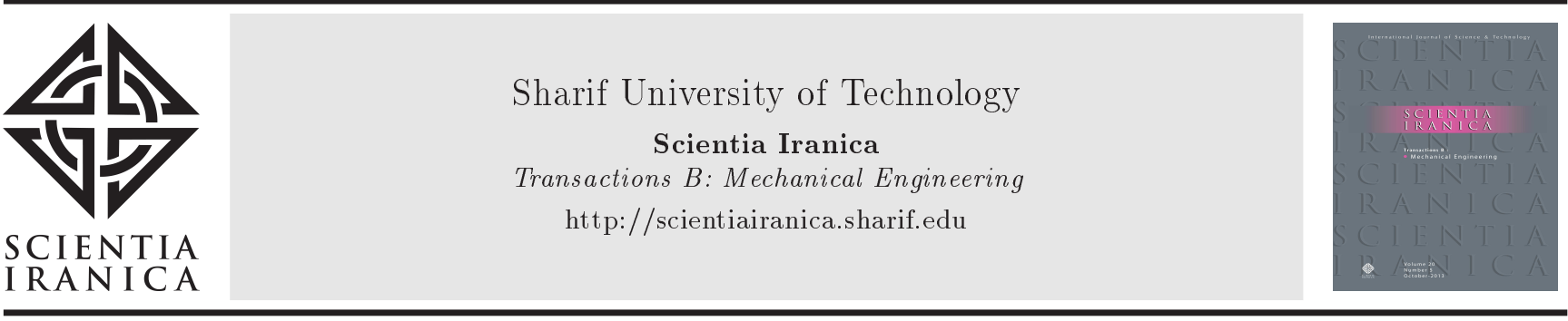

\title{
Numerical solutions of Fourier's law involving fractional derivatives with bi-order
}

\author{
J.F. Gómez-Aguilar ${ }^{\mathrm{a}, *}$, A. Atangana ${ }^{\mathrm{b}}$, and R.F. Escobar-Jiménez ${ }^{\mathrm{c}}$ \\ a. CONACyT-Centro Nacional de Investigación y Desarrollo Tecnológico, Tecnológico Nacional de México, Interior Internado \\ Palmira S/N, Col. Palmira, C.P. 62490, Cuernavaca, Morelos, México. \\ b. Institute for Groundwater Studies, Faculty of Natural and Agricultural Sciences, University of the Free State, Bloemfontein 9300, \\ South Africa. \\ c. Centro Nacional de Investigación y Desarrollo Tecnológico, Tecnológico Nacional de México, Interior Internado Palmira S/N, \\ Col. Palmira, C.P. 62490, Cuernavaca, Morelos, México.
}

Received 22 November 2016; received in revised form 17 February 2017; accepted 31 May 2017

\author{
KEYWORDS \\ Anomalous diffusion; \\ Fractional heat \\ transfer model; \\ Iterative method; \\ Bi-order fractional \\ derivative; \\ Non-Fourier heat \\ conduction.
}

\begin{abstract}
In this paper, we present an alternative representation of the fractional spacetime Fourier's law equation using the concept of derivative with two fractional orders $\alpha$ and $\beta$. The new definitions are based on the concept of power law and the generalized Mittag-Leffler function, where the first fractional order is incorporated into the power law function, and the second fractional order is the generalized Mittag-Leffler function. The new approach is capable of considering media with two different layers, scales, and properties. The generalization of this equation exhibits different cases of anomalous behaviors and Non-Fourier heat conduction processes. Numerical solutions are obtained using an iterative scheme.
\end{abstract}

(C) 2018 Sharif University of Technology. All rights reserved.

\section{Introduction}

Fourier's law combined with the energy conservation principle is the basis for the analysis of most conduction processes [1]. However, the Fourier's equation is not adequate to describe certain processes in some cases. Fractional Calculus (FC) is the generalization of ordinary calculus. In recent years, fractional systems have been considered in many publications, e.g., biomedical, electromagnetism, electrical circuits, and transport phenomena [2-10].

The study of non-Fourier heat conduction processes has attracted much interest in recent years. Mainardi et al. [11] presented the interpretation of the corresponding Green function as a probability density

\footnotetext{
*. Corresponding author.

E-mail address: jgomez@cenidet.edu.mx (J.F. Gómez-Aguilar)
}

doi: $10.24200 /$ sci. 2017.4342 and obtained the fundamental space-time fractional diffusion equation from the standard diffusion equation. Qi and Jiang [12] derived the exact solution to the Cattaneo-Vernotte equation by joint Laplace and Fourier transforms. Liu et al. [13] proposed a new timespace fractional Cattaneo-Christov upper-convective derivative flux heat conduction model. In this work, the space fractional derivative was characterized by the weight coefficient of forward versus backward transition probability. Ezzat et al. [14] presented a new mathematical model of heat conduction; they considered the isotropic generalized thermoelasticity with a threephase lag, and their proposed model was considered as the methodology of FC. Zhao et al. [15] studied the Soret-Dufour effects on Maxwell fluid embedded in Darcy-Boussinesq medium. Other applications of FC in heat conduction are given in [16-21].

The fractional order that appears in the RiemannLiouville or Liouville-Caputo operators can be used to represent some physical parameters. Nevertheless, it is not possible for these operators to be used in order 
to describe the movement of heat via material with different layers, where each layer possesses different materials. To solve the problem, Abdon Atangana [22] introduced fractional operators with two orders in Riemann-Liouville and Liouville-Caputo sense using the concept of fractional derivative with non-local and non-singular kernel. These operators allow for describing problems that are more complex with different layers and different properties, e.g., the problem in the case of thermal conduction where the heat is flowing within a medium with two different properties. In this context, the aim of this contribution is to present an alternative representation of the fractional-time Fourier's law equation using the concept of derivative with two fractional orders $\alpha$ and $\beta$. The fractional orders considered are $n-1<\alpha<n$ and $0<\beta<1$ for the fractional equation.

The paper is organized as follows: Section 2 presents new definitions of fractional operators with biorder; Section 3 discusses the fractional-time Fourier's law equation; Section 4 concludes the paper.

\section{Fractional operators with bi-order}

In the following, some definitions of fractional operators with bi-order are presented [22].

The Atangana-Caputo (AC) definition with biorder $(\alpha, \beta)$ for $f(t)$ is:

$$
\begin{gathered}
{ }_{0}^{A C} D_{t}^{\alpha, \beta} f(t)=\frac{B(\beta)}{n-\beta} \cdot \frac{1}{\Gamma(n-\alpha)} \int_{0}^{t} \frac{d^{n}}{d t^{n}} f(t) \\
(t-\tau)^{1-\alpha-n} E_{\beta}\left\{-\frac{\beta}{n-\beta}(t-\tau)^{\beta+\alpha}\right\} d \tau . \\
n-1<\beta<n, \quad n-1<\alpha<n .
\end{gathered}
$$

The Laplace transform of Eq. (1) produces:

$$
\begin{aligned}
& \mathcal{L}\left[{ }_{0}^{A C} \mathcal{D}_{t}^{\alpha, \beta} f(t)\right](s)=\frac{B(\beta)}{1-\beta} \cdot \frac{1}{\Gamma(1-\alpha)}\{s F(s)-f(0)\} \\
& \mathcal{L}\left\{t^{-\alpha} E_{\beta}\left(-\frac{\beta}{1-\beta} t^{\beta+\alpha}\right\}\right\}
\end{aligned}
$$

where:

$$
\begin{aligned}
\mathcal{L}\left\{t^{-\alpha} E_{\beta}\left(-\frac{\beta}{1-\beta} t^{\beta+\alpha}\right)\right\} & =s^{\alpha-1} \Psi_{1} \\
& \left.\begin{array}{cc}
(1,1),(1-\alpha, \alpha+\beta) ; & -\left(\frac{\beta}{1-\beta} \cdot \frac{1}{s^{\alpha+\beta}}\right)
\end{array}\right],
\end{aligned}
$$

function ${ }_{a} \Psi_{b}$ is the Wright's generalized hypergeometric function [23].
Substituting Wright's function (3) into Eq. (2), we obtain:

$$
\begin{aligned}
& \mathcal{L}\left[{ }_{0}^{A C} \mathcal{D}_{t}^{\alpha, \beta} f(t)\right](s)=\frac{B(\beta)}{1-\beta} \cdot \frac{s}{\Gamma(1-\alpha)}{ }^{2} \Psi_{1}
\end{aligned}
$$

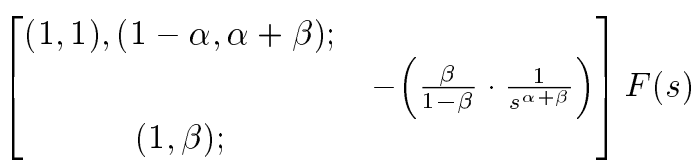

$$
\begin{aligned}
& -\frac{B(\beta)}{1-\beta} \cdot \frac{s^{\alpha-1}}{\Gamma(1-\alpha)}{ }^{2} \Psi_{1}
\end{aligned}
$$

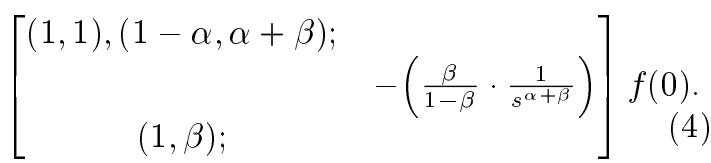

For this operator, the inverse Laplace transform is defined by:

$$
\begin{gathered}
{ }_{0}^{A C} \mathcal{D}_{t}^{\alpha, \beta} f(t)={ }_{0}^{A R} \mathcal{D}_{t}^{\alpha, \beta} f(t)-\frac{B(\beta)}{1-\beta} \cdot \frac{1}{\Gamma(1-\alpha)} \\
\cdot f(0) t^{-\alpha} E_{\beta}\left\{-t^{\beta+\alpha}\right\} .
\end{gathered}
$$

For the fractional operator with bi-order (1), when $\alpha=0$, we recover Atangana-Baleanu fractional derivative in Liouville-Caputo sense (ABC). This operator is defined as follows [24]:

$$
\begin{aligned}
{ }_{0}^{\mathrm{ABC}} \mathcal{D}_{t}^{\beta} f(t) & =\frac{B(\beta)}{1-\beta} \int_{0}^{t} \dot{f}(\tau) E_{\beta}\left[-\frac{\beta}{1-\beta}(t-\tau)^{\beta}\right] d \tau \\
0<\beta & \leq 1
\end{aligned}
$$

where $\frac{d^{\beta}}{d t^{\beta}}={ }_{0}^{\mathrm{ABC}} \mathcal{D}_{t}^{\beta}$ is an ABC fractional derivative with respect to $t$, and $B(\beta)$ is a normalization function that has the same properties as in Caputo and CaputoFabrizio case.

The Laplace transform of Eq. (6) is defined as follows:

$$
\begin{aligned}
\left.\mathcal{L}_{0}^{\mathrm{ABC}} \mathcal{D}_{t}^{\beta} f(t)\right](s) & =\frac{B(\beta)}{1-\beta} \mathcal{L}\left[\int_{a}^{t} \dot{f}(\tau) E_{\beta}\right. \\
& {\left.\left[-\frac{\beta}{1-\beta}(t-\tau)^{\beta}\right] d \tau\right] } \\
& =\frac{B(\beta)}{1-\beta} \frac{s^{\beta} \mathcal{L}[f(t)](s)-s^{\beta-1} f(0)}{s^{\beta}+\frac{\beta}{1-\beta}} .
\end{aligned}
$$

For the fractional operator with bi-order Eq. (1), when $\beta=0$, we recover Liouville-Caputo fractional derivative $(C)$. This operator is defined as follows:

$$
\begin{aligned}
{ }_{0}^{C} \mathcal{D}_{t}^{\alpha} f(t) & =\frac{1}{\Gamma(1-\alpha)} \int_{0}^{t}(t-\tau)^{-\alpha} \frac{d}{d t} f(\tau) d \tau, \\
0<\alpha & \leq 1] .
\end{aligned}
$$




\section{Fourier's law equation}

To keep the dimensionality of the differential equation, new parameters $\sigma_{t}$ and $\sigma_{x}$ are introduced [25]. For the AC fractional derivative with bi-order Eq. (1), we have:

$$
\begin{aligned}
& \frac{d}{d t} \rightarrow \frac{1}{\sigma_{t}^{1-\alpha, \beta}} \cdot{ }_{0}^{A C} \mathcal{D}_{t}^{\alpha, \beta}, \quad 0<\alpha, \beta \leq 1, \\
& \frac{d^{2}}{d x^{2}} \rightarrow \frac{1}{\sigma_{x}^{2(1-\alpha, \beta)}} \cdot{ }_{0}^{A C} \mathcal{D}_{x}^{2(\alpha, \beta)}, \quad 0<\alpha, \beta \leq 1,
\end{aligned}
$$

when $\alpha=0$ in Eqs. (9) and (10), we obtain the fractional operator of type $\mathrm{ABC}$ (6) as follows:

$$
\begin{aligned}
& \frac{d}{d t} \rightarrow \frac{1}{\sigma_{t}^{1-\beta}} \cdot{ }_{0}^{\mathrm{ABC}} \mathcal{D}_{t}^{\beta}, \quad 0<\beta \leq 1, \\
& \frac{d^{2}}{d x^{2}} \rightarrow \frac{1}{\sigma_{x}^{2(1-\beta)}} \cdot{ }_{0}^{\mathrm{ABC}} \mathcal{D}_{x}^{2 \beta}, \quad 0<\beta \leq 1 .
\end{aligned}
$$

For all cases, $\sigma_{t}$ has the dimension of time, and $\sigma_{x}$ has the dimension of length. These parameters are associated with the temporal and spatial components in the system [25], when $(\alpha=\beta=1)$ and $(\beta=1)$, respectively; the expressions above are recovered in the traditional sense. From now on, this idea will be appliedto the fractional Fourier's law.

The Fourier's law is described by the classical parabolic equation:

$$
\chi \frac{\partial^{2} T(x, t)}{\partial x^{2}}-\frac{\partial T(x, t)}{\partial t}=0
$$

where $\chi=\frac{k}{\rho C_{p}}, \chi$ is the thermal diffusivity, $k$ is the thermal conductivity, $\rho$ is density, $C_{p}$ is the specific heat capacity, and $T$ is the temperature. conduction in a planar medium with constant properties.

\subsection{Fractional time Fourier's law equation}

Considering Eq. (13) and assuming that the time derivative is fractional and the space derivative is ordinary, the temporal fractional equation will be as follows:

$$
{ }_{0}^{A C} \mathcal{D}_{t}^{\alpha, \beta} T(x, t)-\chi \frac{\partial^{2} T(x, t)}{\partial x^{2}}=0 .
$$

A particular solution to Eq. (13) can be found in the following form:

$$
T(x, t)=T_{0} \cdot e^{-i \tilde{k} x} u(t)
$$

Substituting Eq. (15) into Eq. (14) and considering Eq. (9), we obtain:

$$
{ }_{0}^{A C} \mathcal{D}_{t}^{\alpha, \beta} u(t)+\tilde{\omega} u(t)=0,
$$

where $\tilde{\omega}=\omega \sigma_{t}^{1-\alpha, \beta}$ and $\omega=\chi \tilde{k}^{2}$ are the angular frequencies.

The numerical approximation of Eq. (16) is given by:

$$
\begin{aligned}
& \frac{B(\beta)}{1-\beta} \cdot \frac{1}{\Gamma(1-\alpha)} \int_{0}^{t} \frac{d u(\tau)}{d \tau}(t-\tau)^{-\alpha} \\
& E_{\beta}\left\{-\frac{\beta}{1-\beta}(t-\tau)^{\beta+\alpha}\right\} d \tau+\tilde{\omega} u(t)=0
\end{aligned}
$$

$$
\begin{gathered}
\frac{B(\beta)}{1-\beta} \cdot \frac{1}{\Gamma(1-\alpha)} \int_{0}^{t} \frac{u(t)-u(t+\Delta t)}{2 \Delta t}(t-\tau)^{-\alpha} E_{\beta} \\
\left\{-\frac{\beta}{1-\beta}(t-\tau)^{\beta+\alpha}\right\} d \tau+\tilde{\omega} u(t)=0 \\
\frac{B(\beta)}{1-\beta} \cdot \frac{1}{\Gamma(1-\alpha)} \sum_{i=0}^{n} \int_{t_{i}}^{t_{i+1}} \frac{u\left(t_{i+1}\right)-u\left(t_{i}\right)}{2 \Delta t} \\
\left(t_{n}-\tau\right)^{-\alpha} E_{\beta}\left\{-\frac{\beta}{1-\beta}\left(t_{n}-\tau\right)^{\beta+\alpha}\right\} d \tau \\
+\tilde{\omega}\left[\frac{u\left(t_{n+1}\right)-u\left(t_{n}\right)}{2}\right]=0
\end{gathered}
$$

$$
\begin{gathered}
\frac{B(\beta)}{1-\beta} \cdot \frac{1}{\Gamma(1-\alpha)} \sum_{i=0}^{n} \frac{u\left(t_{i+1}\right)-u\left(t_{i}\right)}{2 \Delta t} \int_{t_{n}-t_{i}}^{t_{n}-t_{i}+1} \nu^{-\alpha} \\
E_{\beta}\left\{-\frac{\beta}{1-\beta} \nu^{\beta+\alpha}\right\} d \nu+\tilde{\omega}\left[\frac{u\left(t_{n+1}\right)-u\left(t_{n}\right)}{2}\right]=0
\end{gathered}
$$

thus:

$$
\begin{aligned}
u(t)= & \frac{B(\beta)}{1-\beta} \cdot \frac{1}{\Gamma(1-\alpha)} \sum_{i=0}^{n} \frac{u^{i+1}-u^{i}}{2 \Delta t} \\
& {\left[\left(t_{n}-t_{i+1}\right)^{1-\alpha} E_{\beta, 2-\alpha}\right.} \\
& \left\{-\frac{\beta}{1-\beta}\left(t_{n}-t_{i+1}\right)^{\beta+\alpha}\right\} \\
& -\left(t_{n}-t_{i}\right)^{1-\alpha} E_{\beta, 2-\alpha}\{ \\
& \left.\left.-\frac{\beta}{1-\beta}\left(t_{n}-t_{i+1}\right)^{\beta+\alpha}\right\}\right] \\
& +\tilde{\omega}\left[\frac{u\left(t_{n+1}\right)-u\left(t_{n}\right)}{2}\right]
\end{aligned}
$$

where $E_{\beta}$ is the Mittag-Leffler function. 

follows:

The particular solution to Eq. (16) is written as

$$
\begin{aligned}
T(x, t)= & T_{0} \cdot e^{-i \tilde{k} x}\left[\frac{B(\beta)}{1-\beta} \cdot \frac{1}{\Gamma(1-\alpha)} \sum_{i=0}^{n} \frac{u^{i+1}-u^{i}}{2 \Delta t}\right. \\
& {\left[\left(t_{n}-t_{i+1}\right)^{1-\alpha} \cdot E_{\beta, 2-\alpha}\right.} \\
& \left\{-\frac{\beta}{1-\beta}\left(t_{n}-t_{i+1}\right)^{\beta+\alpha}\right\} \\
& -\left(t_{n}-t_{i}\right)^{1-\alpha} E_{\beta, 2-\alpha} \\
& \left.\left\{-\frac{\beta}{1-\beta}\left(t_{n}-t_{i+1}\right)^{\beta+\alpha}\right\}\right] \\
& \left.+\tilde{\omega}\left[\frac{u\left(t_{n+1}\right)-u\left(t_{n}\right)}{2}\right]\right] .
\end{aligned}
$$

Eq. (19) represents the time thermal diffusion.

If $\alpha=0$ in Eq. (16), we recover $\mathrm{ABC}$ fractional derivative Eq. (6); from Eq. (14), we have:

$$
{ }_{0}^{\mathrm{ABC}} \mathcal{D}_{t}^{\beta} T(x, t)-\chi \frac{\partial^{2} T(x, t)}{\partial x^{2}}=0 .
$$

A particular solution to this equation can be found in the form of Eq. (15), and by substituting Eq. (15) into Eq. (20) and considering Eq. (11), we obtain:

$$
{ }_{0}^{\mathrm{ABC}} \mathcal{D}_{t}^{\alpha, \beta} u(t)+\tilde{\omega} u(t)=0
$$

where $\tilde{\omega}=\omega \sigma_{t}^{1-\beta}$ and $\omega=\chi \tilde{k}^{2}$.

Applying Laplace transforms (7) to (21) and considering $U(0)=u_{0}$ yield the following expression:

$$
\begin{aligned}
U(s)= & \frac{(1-\beta) s^{\beta-1}}{s^{\beta}[B(\beta)+\tilde{\omega}(1-\beta)]}+\frac{B(\beta) u_{0}}{B(\beta)+\tilde{\omega}(1-\beta)} \\
& \cdot \frac{s^{\beta-1}}{s^{\beta}+\frac{\tilde{\omega} \beta}{B(\beta)+\tilde{\omega}(1-\beta)}} .
\end{aligned}
$$

Taking the inverse Laplace transform of Eq. (22), we obtain the following particular solution of the Eq. (21):

$$
\begin{aligned}
T(x, t)= & T_{0} \cdot e^{-i \tilde{k} x}\left\{\frac{1-\beta}{B(\beta)+\tilde{\omega}(1-\beta)}\right. \\
& \cdot E_{\beta, 1}\left[-\left(\frac{\tilde{\omega} \beta}{B(\beta)+\tilde{\omega}(1-\beta)}\right) t^{\beta}\right] \\
& +\frac{B(\beta) u_{0}}{B(\beta)+\tilde{\omega}(1-\beta)} \\
& \left.\cdot E_{\beta, 1}\left[-\left(\frac{\tilde{\omega} \beta}{B(\beta)+\tilde{\omega}(1-\beta)}\right) t^{\beta}\right]\right\}
\end{aligned}
$$

where $E_{\beta}$ is the Mittag-Leffler function. In this case, when $(\beta=1)$, we have:

$$
\tilde{T}(x, t)=\Re\left[\tilde{T}_{0} \cdot e^{-\omega t-i \tilde{k} x}\right],
$$

where $\Re$ indicates the real part. Equation (24) represents the classical case for the time Fourier's law equation.

Figure 1 shows numerical simulations for temperature, $T(x, t)$, considering different values of $\alpha$ and $\beta$, in Eq. (19), arbitrarily chosen.

Figure 2 shows numerical simulations for temperature, $T(x, t)$, considering different values of $\beta$, in Eq. (23), arbitrarily chosen.

Considering, $\tilde{\omega}=\chi \tilde{k}^{2} \sigma_{t}^{1-\beta}$ and $1 / \chi$ as the reciprocal of the time constant or thermal diffusion coefficient, we have:

$$
\tilde{\omega}=\tilde{k}^{2}\left(\frac{\sigma_{t}^{1-\beta}}{\chi}\right)=\tilde{k}^{2}\left(\frac{1}{\tau_{\beta}}\right),
$$

where if $\tau_{\beta}=\chi \sigma_{t}^{\beta-1}$, it can be called fractional time constant, and when $\beta=1$, it is the classical time constant; $\tilde{\omega}$ is the angular frequency in the medium in the presence of fractional time components, $\tilde{k}$ is the wave number, and $1 / \chi$ is the time constant of the system. Substituting Eq. (25) into Eq. (23), we obtain:

$$
\begin{aligned}
T(x, t)= & T_{0} \cdot e^{-i k x}\left\{\frac{1-\beta}{B(\beta)+\left(\frac{\tilde{k}^{2}}{\tau_{\beta}}\right)(1-\beta)}\right. \\
& \cdot E_{\beta, 1}\left[-\left(\frac{\left(\frac{\tilde{k}^{2}}{\tau_{\beta}}\right) \beta}{B(\beta)+\left(\frac{\tilde{k}^{2}}{\tau_{\beta}}\right)(1-\beta)}\right) t^{\beta}\right] \\
& +\frac{B(\beta) u_{0}}{B(\beta)+\left(\frac{\tilde{k}^{2}}{\tau_{\beta}}\right)(1-\beta)} \\
& \left.\cdot E_{\beta, 1}\left[-\left(\frac{\left(\frac{\tilde{k}^{2}}{\tau_{\beta}}\right) \beta}{B(\beta)+\left(\frac{\tilde{k}^{2}}{\tau_{\beta}}\right)(1-\beta)}\right) t^{\beta}\right]\right\} .
\end{aligned}
$$

Figure 3 shows the simulation results of Eq. (26) using fractional exponents $\beta=1, \beta=0.95, \beta=0.9$, $\beta=0.85$, and $\beta=0.80$, respectively.

Table 1 shows different values of the thermal diffusion when $\beta$ changes from $\beta=1, \beta=0.95, \beta=$ 0.90 to $\beta=0.85$, respectively, when $\beta<1$, the thermal

Table 1. Thermal diffusion versus Constant time $\tau_{\beta}$.

\begin{tabular}{ccc}
\hline $\boldsymbol{\beta}$ & Constant time $\left(\boldsymbol{\tau}_{\boldsymbol{\beta}}\right)$ & Thermal diffusion \\
\hline 1 & 1 & 0.63 \\
0.95 & 0.9376 & 0.63 \\
0.90 & 0.9216 & 0.63 \\
0.85 & 0.9143 & 0.63 \\
\hline
\end{tabular}




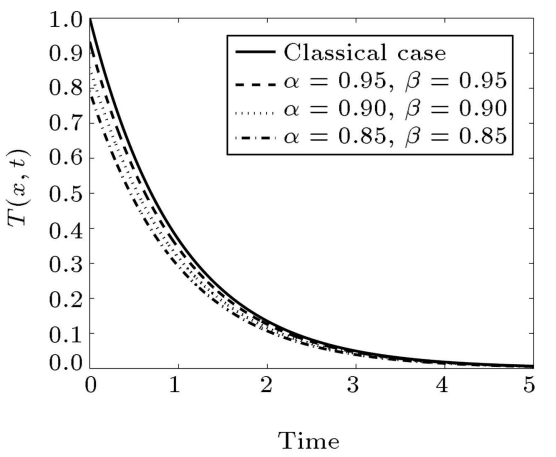

(a)

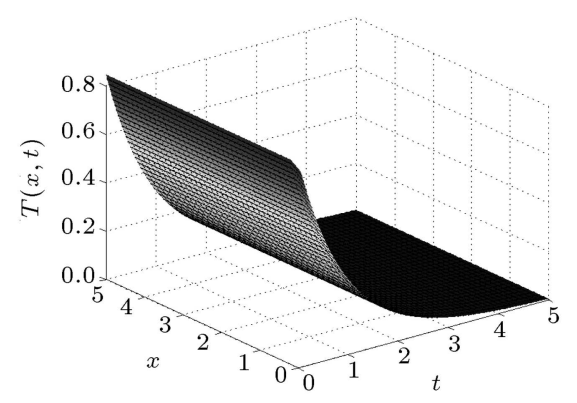

(c)

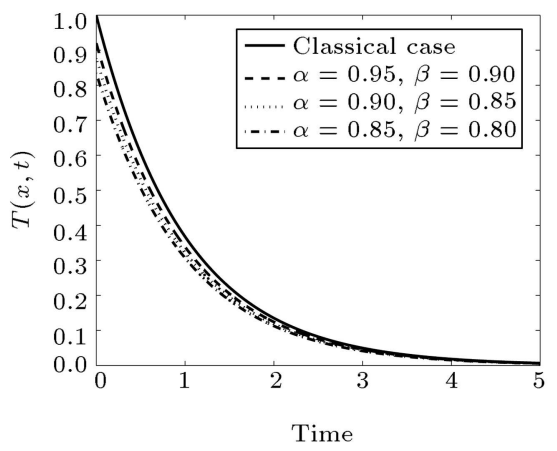

(b)

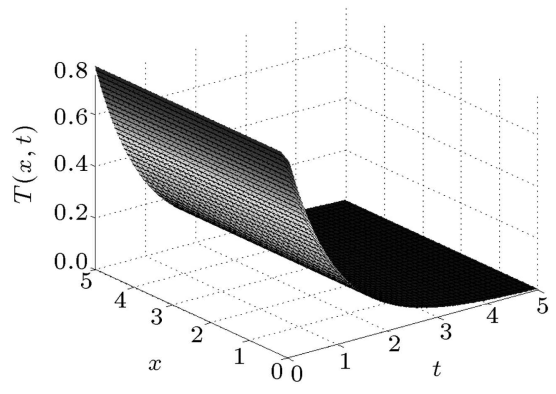

(d)

Figure 1. Thermal diffusion for different values of $\alpha$ and $\beta$ arbitrarily chosen, using numerical evaluation of Eq. (19): (a) $\alpha$ and $\beta$ take the same value, (b) $\alpha$ and $\beta$ take different values, (c) $\alpha=0.90$ and $\beta=0.85$, and (d) $\alpha=0.85$ and $\beta=0.80$.

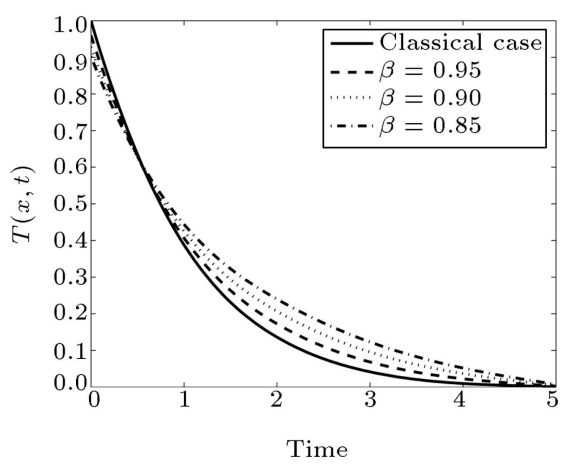

(a)

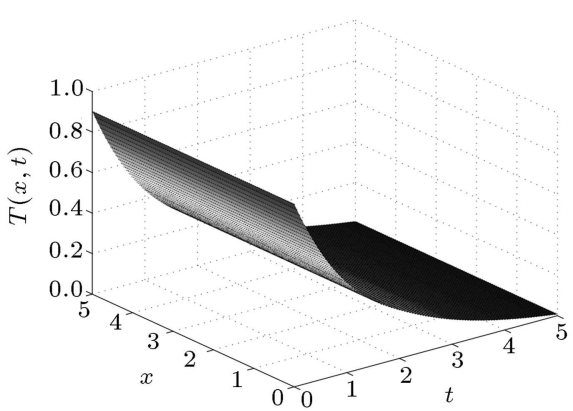

(b)

Figure 2. Thermal diffusion for different values of $\beta$ arbitrarily chosen, using numerical evaluation of Eq. (23): (a) $\beta$ takes different values, and (b) $\beta=0.85$.

diffusion occurs in less time than the ordinary thermal diffusion. This phenomenon indicates the change of the medium properties, and the system presents dissipative effects [11].

\subsection{Fractional space Fourier's law equation}

Considering Eq. (13) and assuming that the space derivative is fractional and the time derivative is ordinary, the spatial fractional equation will be as follows:

$$
{ }_{0}^{A C} \mathcal{D}_{x}^{2(\alpha, \beta)} T(x, t)-\frac{1}{\chi} \frac{\partial T(x, t)}{\partial t}=0 .
$$

A particular solution to Eq. (27) can be found in the following form:

$$
T(x, t)=T_{0} \cdot e^{-\omega t} u(x) .
$$

Substituting Eq. (28) into Eq. (27) and considering Eq. (10), we obtain:

$$
{ }_{0}^{A C} \mathcal{D}_{x}^{2(\alpha, \beta)} u(x)+\tilde{k}^{2} u(x)=0
$$

where $\tilde{k}^{2}=k^{2} \sigma_{x}^{2(1-\alpha, \beta)}$ is the fractional wave number with bi-order and $k=\frac{\omega}{\chi}$ is the classical wave number. The numerical approximation of Eq. (29) is given by: 


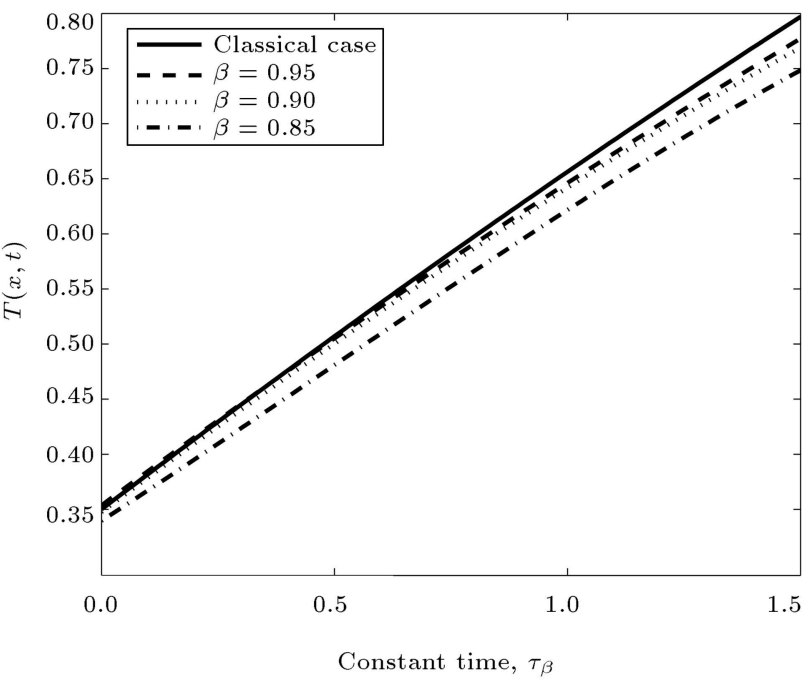

Figure 3. Thermal diffusion versus constant time, using exponent $\beta=1, \tau_{\beta}=0.63$ located in $t=1 \mathrm{~s}, \beta=0.95$; $\tau_{\beta}=0.63$ located in $t=1.033 \mathrm{~s}, \beta=0.90 ; \tau_{\beta}=0.63$ located in $t=1.037 \mathrm{~s}, \beta=0.85 ; \tau_{\beta}=0.63$ located in $t=1.041 \mathrm{~s}$ and $\beta=0.80 ; \tau_{\beta}=0.63$ located in $t=1.044 \mathrm{~s}$.

$$
\begin{aligned}
& \frac{B(\beta)}{2-\beta} \cdot \frac{1}{\Gamma(2-\alpha)} \int_{0}^{x} \frac{d^{2} u(\eta)}{d \eta^{2}}(x-\eta)^{-\alpha} E_{2 \beta} \\
& \left\{-\frac{\beta}{2-\beta}(x-\eta)^{2(\beta+\alpha)}\right\} d \eta+\tilde{k}^{2} u(x)=0, \\
& \frac{B(\beta)}{2-\beta} \cdot \frac{1}{\Gamma(2-\alpha)} \int_{0}^{x} \frac{u(x)-u(x+\Delta x)}{2 \Delta x} \\
& (x-\eta)^{-\alpha} E_{2 \beta}\left\{-\frac{\beta}{1-\beta}(x-\eta)^{2(\beta+\alpha)}\right\} d \eta \\
& +\tilde{k}^{2} u(x)=0,
\end{aligned}
$$$$
\frac{B(\beta)}{2-\beta} \cdot \frac{1}{\Gamma(2-\alpha)} \sum_{i=0}^{n}
$$$$
\int_{x_{i}}^{x_{i+1}} \frac{u\left(x_{i+1}\right)-2 u\left(x_{i}\right)+u\left(x_{i-1}\right)}{2(\Delta x)^{2}}
$$$$
\left(x_{n}-\eta\right)^{-\alpha} \cdot E_{2 \beta}\left\{-\frac{\beta}{2-\beta}\left(x_{n}-\eta\right)^{2(\beta+\alpha)}\right\} d \eta
$$$$
+\tilde{k}^{2}\left[\frac{u\left(x_{n+1}\right)-u\left(x_{n}\right)}{2}\right]=0,
$$$$
\frac{B(\beta)}{2-\beta} \cdot \frac{1}{\Gamma(2-\alpha)} \sum_{i=0}^{n} \frac{u\left(x_{i+1}\right)-2 u\left(x_{i}\right)+u\left(x_{i-1}\right)}{2(\Delta x)^{2}}
$$$$
\int_{x_{n}-x_{i}}^{x_{n}-x_{i}+1} \nu^{-\alpha}\left(x_{n}-\eta\right)^{-\alpha}
$$$$
E_{2 \beta}\left\{-\frac{\beta}{2-\beta}\left(x_{n}-x_{i+1}\right)\right\} d \eta
$$

$$
+\tilde{k}^{2}\left[\frac{u\left(x_{n+1}\right)-u\left(x_{n}\right)}{2}\right]=0
$$

thus:

$$
\begin{aligned}
u(x)= & \frac{B(\beta)}{2-\beta} \cdot \frac{1}{\Gamma(2-\alpha)} \\
& \sum_{i=0}^{n} \frac{u\left(x_{i+1}\right)-2 u\left(x_{i}\right)+u\left(x_{i-1}\right)}{2(\Delta x)^{2}} \\
& {\left[\left(x_{n}-x_{i+1}\right)^{1-\alpha} \cdot E_{2 \beta, 2-\alpha}\right.} \\
& \left\{-\frac{\beta}{2-\beta}\left(x_{n}-x_{i+1}\right)^{2(\beta+\alpha)}\right\} \\
& -\left(x_{n}-x_{i}\right)^{1-\alpha} E_{2 \beta, 2-\alpha} \\
& \left.\left\{-\frac{\beta}{2-\beta}\left(x_{n}-x_{i+1}\right)^{2(\beta+\alpha)}\right\}\right] \\
& +\tilde{k}^{2}\left[\frac{u\left(x_{n+1}\right)-u\left(x_{n}\right)}{2}\right]
\end{aligned}
$$

where $E_{2 \beta}$ is the Mittag-Leffler function.

The particular solution to Eq. (28) is written as follows:

$$
\begin{aligned}
T(x, t)= & T_{0} \cdot e^{-\omega t}\left[\frac{B(\beta)}{2-\beta}\right. \\
& \cdot \frac{1}{\Gamma(2-\alpha)} \sum_{i=0}^{n} \frac{u\left(x_{i+1}\right)-2 u\left(x_{i}\right)+u\left(x_{i-1}\right)}{2(\Delta x)^{2}} \\
& {\left[\left(x_{n}-x_{i+1}\right)^{1-\alpha} \cdot E_{2 \beta, 2-\alpha}\right.} \\
& \left\{-\frac{\beta}{2-\beta}\left(x_{n}-x_{i+1}\right)^{2(\beta+\alpha)}\right\} \\
& -\left(x_{n}-x_{i}\right)^{1-\alpha} \cdot E_{2 \beta, 2-\alpha} \\
& \left.\left\{-\frac{\beta}{2-\beta}\left(x_{n}-x_{i+1}\right)^{2(\beta+\alpha)}\right\}\right] \\
& \left.+\tilde{k}^{2}\left[\frac{u\left(x_{n+1}\right)-u\left(x_{n}\right)}{2}\right]\right]
\end{aligned}
$$

Eq. (31) represents the thermal diffusion with spatialdecaying amplitude with respect to space $x$.

If $\alpha=0$ in Eq. (29), we recover ABC fractional derivative (6); from Eq. (27), we have:

$$
{ }_{0}^{\mathrm{ABC}} \mathcal{D}_{t}^{\beta} T(x, t)-\chi \frac{\partial^{2} T(x, t)}{\partial x^{2}}=0 .
$$


A particular solution to this equation can be found in the form of Eq. (28); substituting Eq. (28) into Eq. (32) and considering Eq. (12), we obtain:

$$
{ }_{0}^{\mathrm{ABC}} \mathcal{D}_{x}^{2 \beta} u(x)+\tilde{k}^{2} u(x)=0,
$$

where, $\tilde{k}^{2}=k^{2} \sigma_{x}^{2(1-\beta)}$ is the fractional wave number and $k=\frac{\omega}{\chi}$ is the classical wave number.

Applying Laplace transform (8) to (33) and considering $u(0)=u_{0}$ and $\dot{u}(0)=0$ yield the following expression:

$$
\begin{aligned}
U(s)= & \frac{1}{B(\beta)^{2}+\tilde{k}^{2}(1-\beta)^{2}} \\
& {\left[\frac{(1-\beta)^{2} s^{2 \beta-1}}{\left(s^{\beta}+\frac{i \tilde{k} \beta}{B(\beta)+i \tilde{k}(1-\beta)}\right)\left(s^{\beta}-\frac{i \tilde{k} \beta}{B(\beta)-i \tilde{k}(1-\beta)}\right)}\right.} \\
& +\frac{2 \beta(1-\beta) s^{\beta-1}}{\left(s^{\beta}+\frac{i \tilde{k} \beta}{B(\beta)+i \tilde{k}(1-\beta)}\right)\left(s^{\beta}-\frac{i \tilde{k} \beta}{B(\beta)-i \tilde{k}(1-\beta)}\right)} \\
& \left.+\frac{\beta^{2} s^{-1}}{\left(s^{\beta}+\frac{i \tilde{k} \beta}{B(\beta)+i \tilde{k}(1-\beta)}\right)\left(s^{\beta}-\frac{i \tilde{k} \beta}{B(\beta)-i \tilde{k}(1-\beta)}\right)}\right] \\
& +\frac{B(\beta)^{2} u_{0}}{B(\beta)^{2}+\tilde{k}^{2}(1-\beta)^{2}} \\
& {\left[\frac{s^{2 \beta-1}}{\left(s^{\beta}+\frac{i \tilde{k} \beta}{B(\beta)+i \tilde{k}(1-\beta)}\right)\left(s^{\beta}-\frac{i \tilde{k} \beta}{B(\beta)-i \tilde{k}(1-\beta)}\right)}\right] . }
\end{aligned}
$$

Taking the inverse Laplace transform of Eq. (34), we obtain the following particular solution to Eq. (33):

$$
\begin{aligned}
T(x, t)= & T_{0} \cdot e^{-\omega t}\left[\frac{(1-\beta)^{2}}{B(\beta)^{2}+\tilde{k}^{2}(1-\beta)^{2}}\right. \\
& \cdot \frac{a E_{\beta, 1}\left(a x^{\beta}\right)+b E_{\beta, 1}\left(-b x^{\beta}\right)}{a+b} \\
& +\frac{2 \beta(1-\beta)}{B(\beta)^{2}+\tilde{k}^{2}(1-\beta)^{2}} \\
& \cdot \frac{a E_{\beta, \beta+1}\left(a x^{\beta}\right)+b E_{\beta, \beta+1}\left(-b x^{\beta}\right)}{a+b} \cdot x^{\beta} \\
& +\frac{\beta^{2}}{B(\beta)^{2}+\tilde{k}^{2}(1-\beta)^{2}} \\
& \cdot \frac{a E_{\beta, 2 \beta+1}\left(a x^{\beta}\right)+b E_{\beta, 2 \beta+1}\left(-b x^{\beta}\right)}{a+b} \cdot x^{2 \beta}
\end{aligned}
$$

$$
\begin{aligned}
& +\frac{B(\beta) u_{0}}{B(\beta)^{2}+\tilde{k}^{2}(1-\beta)^{2}} \\
& \left.\cdot \frac{a E_{\beta, 1}\left(a x^{\beta}\right)+b E_{\beta, 1}\left(-b x^{\beta}\right)}{a+b}\right],
\end{aligned}
$$

where $a=\frac{i \tilde{k} \beta}{B(\beta)+i \tilde{k}(1-\beta)}, b=\frac{i \tilde{k} \beta}{B(\beta)-i \tilde{k}(1-\beta)}$ and $E_{\beta}$ is the Mittag-Leffler function.

In this case, when $(\beta=1)$, we have:

$$
\tilde{T}(x, t)=\tilde{T}_{0} \cdot e^{-\omega t} \cos (k x) .
$$

Eq. (36) represents the classical case for the space Fourier's law equation.

Figure 4 shows the numerical simulations for temperature, $T(x, t)$, considering different values of $\alpha$ and $\beta$, in Eq. (31), arbitrarily chosen.

Figure 5 shows numerical simulations for temperature, $T(x, t)$, considering different values of $\beta$, in Eq. (23), arbitrarily chosen.

When fractional orders $\alpha, \beta$ change from $\alpha, \beta \in$ $[0.85 ; 1]$, the simulated fractional diffusion occurs in greater time than the simulated ordinary diffusion. This phenomenon indicates the change of the medium properties, different from the ideal properties presented in Eq. (13). The velocity of the concentration wave through a medium is determined by the inertia and the elasticity of the medium. Usually this dissipation is known as internal friction [26].

\subsection{Fractional space-time Fourier's law equation}

Now, considering Eq. (13) and assuming the space time derivatives as fractional, the order of space-time fractional differential equation is $0<\alpha, \beta \leq 1$ and $0<\gamma, \delta \leq 1$; the space-time fractional equation is as follows:

$$
{ }_{0}^{A C} \mathcal{D}_{x}^{2(\alpha, \beta)} T(x, t)-\frac{1}{\chi^{A}{ }^{A C}} \mathcal{D}_{t}^{(\gamma, \delta)} T(x, t)=0 .
$$

The numerical approximation of Eq. (37) is given by:

$$
\begin{aligned}
& T(x, t)=A \cdot\left[\frac{B(\beta)}{2-\beta}\right. \\
& \cdot \frac{1}{\Gamma(2-\alpha)} \sum_{i=0}^{n} \frac{u\left(x_{i+1}\right)-2 u\left(x_{i}\right)+u\left(x_{i-1}\right)}{2(\Delta x)^{2}} \\
& \quad\left[\left(x_{n}-x_{i+1}\right)^{1-\alpha} \cdot E_{2 \beta, 2-\alpha}\right. \\
& \quad\left\{-\frac{\beta}{2-\beta}\left(x_{n}-x_{i+1}\right)^{2(\beta+\alpha)}\right\} \\
& -\left(x_{n}-x_{i}\right)^{1-\alpha} \cdot E_{2 \beta, 2-\alpha}
\end{aligned}
$$




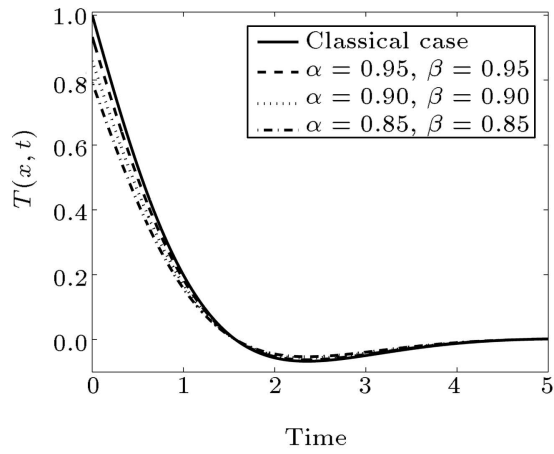

(a)

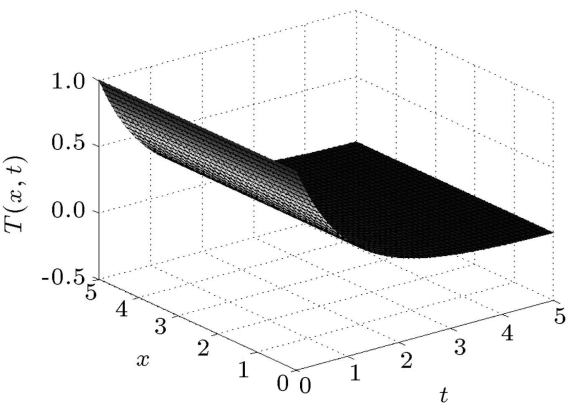

(c)

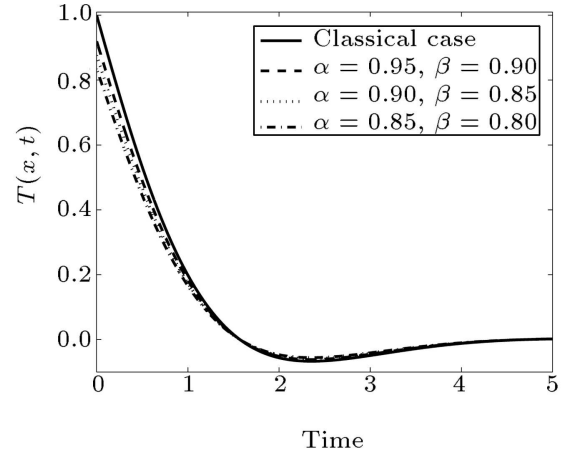

(b)

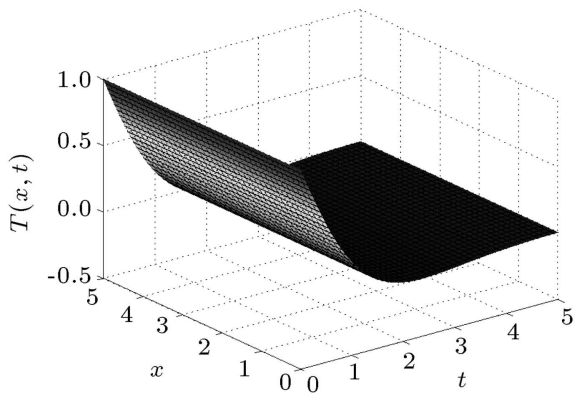

(d)

Figure 4. Thermal diffusion for different values of $\alpha$ and $\beta$ arbitrarily chosen, using numerical evaluation of Eq. (32): (a) $\alpha$ and $\beta$ take the same value, (b) $\alpha$ and $\beta$ take different values, (c) $\alpha=0.90$ and $\beta=0.85$, and (d) $\alpha=0.85$ and $\beta=0.80$.

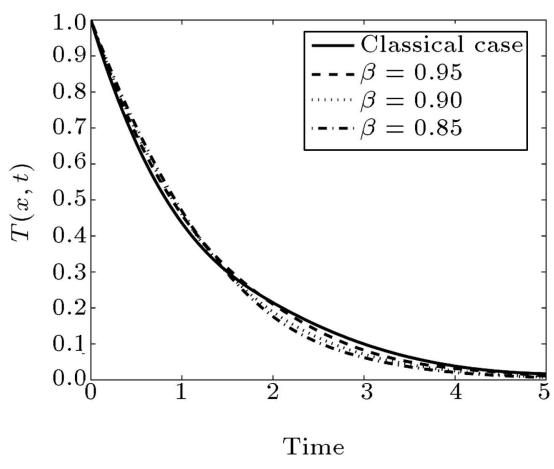

(a)

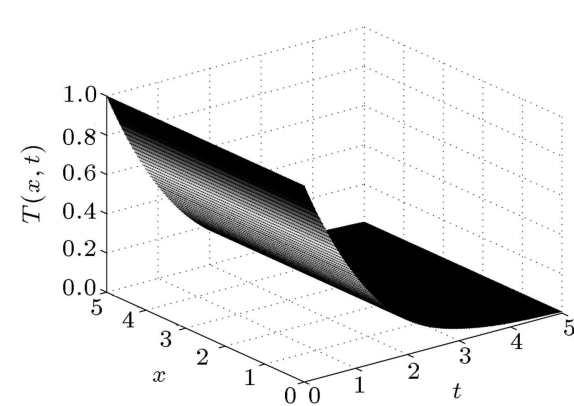

(b)

Figure 5. Thermal diffusion for different values of $\beta$ arbitrarily chosen, using numerical evaluation of Eq. (23): (a) $\beta$ takes different values, and (b) $\beta=0.85$.

$$
\begin{aligned}
& \left.\left.\left\{-\frac{\beta}{2-\beta}\left(x_{n}-x_{i+1}\right)^{2(\beta+\alpha)}\right\}\right]\right] \\
& \cdot\left[\frac{B(\beta)}{1-\delta} \cdot \frac{1}{\Gamma(1-\gamma)}\right. \\
& \sum_{k=0}^{n} \frac{u^{k+1}-u^{k}}{2 \Delta t}\left[\left(t_{n}-t_{k+1}\right)^{1-\gamma} E_{\delta, 2-\gamma}\right. \\
& \left\{-\frac{\delta}{1-\delta}\left(t_{n}-t_{k+1}\right)^{\delta+\gamma}\right\}
\end{aligned}
$$

$$
\begin{aligned}
& -\left(t_{n}-t_{i}\right)^{1-\gamma} E_{\delta, 2-\gamma} \\
& \left.\left.\left\{-\frac{\delta}{1-\delta}\left(t_{n}-t_{k+1}\right)^{\delta+\gamma}\right\}\right]\right]
\end{aligned}
$$

where $A$ is a constant.

Figure 6 shows the simulations where the fractional time and the spatial fractional derivatives are taken at the same time for different particular cases of $(\alpha, \beta)$ and $(\gamma, \delta)$. These figures show different behaviors of anomalous thermal diffusion; when the fractional orders are less than 1, the thermal diffusion is slower 


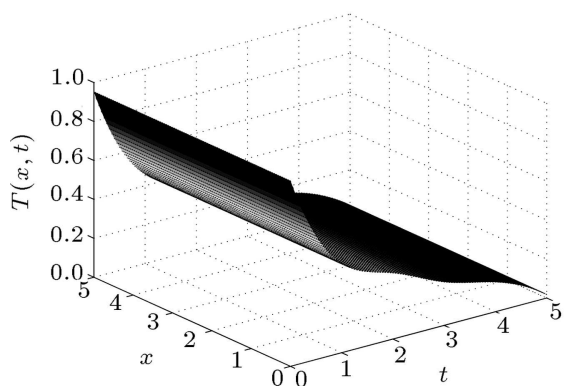

(a)

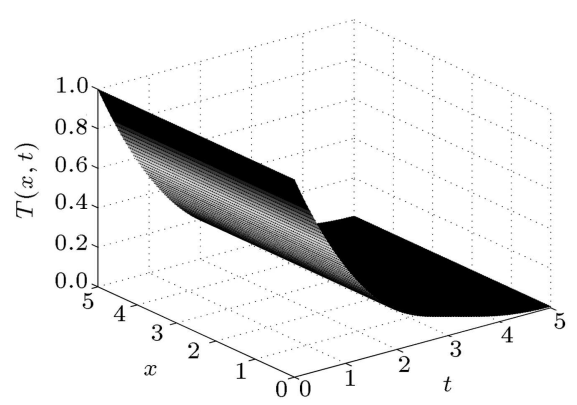

(c)

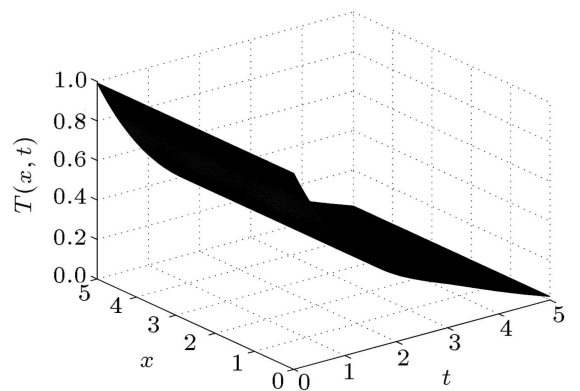

(b)

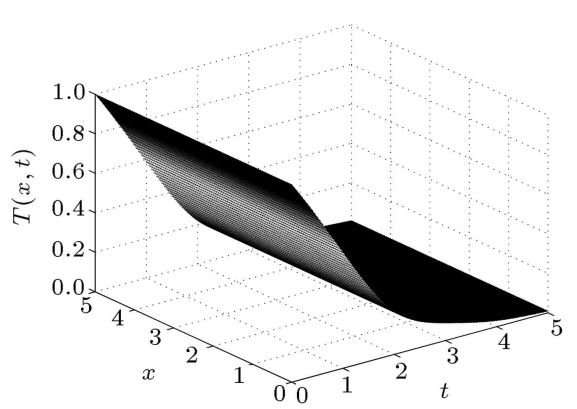

(d)

Figure 6. Thermal diffusion for different values of $(\alpha, \beta)$ and $(\gamma, \delta)$ arbitrarily chosen, using numerical evaluation of Eq. (38): (a) $\alpha=1, \beta=0.9$ and $\gamma=0.95, \delta=0.9$, (b) $\alpha=0.9, \beta=1$ and $\gamma=0.9, \delta=0.95$, and (c) $\alpha=0.95, \beta=0.85$ and $\gamma=0.85, \delta=0.9$, and (d) $\alpha=0.85, \beta=0.9$ and $\gamma=0.8, \delta=0.85$.

(thermal subdiffusion). These cases can represent a medium with two different properties represented by fractional bi-order $\alpha$ and $\beta$.

\section{Conclusion}

In this paper, we presented an alternative representation of the fractional-time Fourier's law equation using the concept of derivative with two fractional orders $\alpha$ and $\beta$. The new approach is capable of considering media with different properties represented by fractional bi-order $\alpha$ and $\beta$. This is the case, for instance, in thermal conduction for a reaction diffusion within a medium with two different layers and properties. These novel fractional operators allow studying the heat transfer through a material with different scales or heterogeneous media. Our results indicate that fractional bi-order $\alpha$ and $\beta$ have an important influence on the temperature.

The motivation of this study comes from the fact that it is possible to find in nature some systems with different material layers, where each layer possesses different materials, e.g., in thermal conduction where the heat is flowing within a medium with two different properties. These types of problems cannot be portrayed with the existing derivatives and fractional order, which are based on the power law. For this reason, the Atangana derivative uses a kernel that is more powerful than $x^{-\beta}$, and the generalized MittagLeffler function is combined with a power law.

In the case where the space-time fractional differential equation is considered, Eq. (38) shows the numerical solution to the Fourier's law equation using the concept of derivative with two fractional orders $(\alpha, \beta)$ and $(\gamma, \delta)$ for space and time, respectively. Different values of $\alpha$ and $\beta$ or $\gamma$ and $\delta$ represent intermediate states between conservative and dissipative systems, which present anomalous relaxations. This combination of stored and dissipated energies is conveniently based on the representation of linear thermoviscoelasticity theory [27].

It is suggested that this derivative can be used to model more complex problems found in nature, groundwater studies, the phenomena of heat transfer from continuous media to discontinuous media, thermal convection of non-Fourier fluids, the non-Newtonian effects in thermal convection, relaxing gas dynamics, irreversible thermodynamics, thermoelasticity, orthodox viscoelastic materials and for the study of systems with heterogeneous media.

\section{Nomenclature}

$\alpha \quad$ Thermal diffusivity $\left(\mathrm{m}^{2} / \mathrm{s}\right)$ 
$\chi \quad$ Thermal conductivity $(\mathrm{W} / \mathrm{mK})$

$\rho \quad$ Density $\left(\mathrm{kg} / \mathrm{m}^{3}\right)$

$C_{p} \quad$ Specific heat capacity $(\mathrm{J} / \mathrm{kgK})$

\section{Acknowledgments}

Abdon Atangana would like to thank NRF for their support. José Francisco Gómez Aguilar acknowledges the support provided by CONACyT: Cátedras CONACyT para jóvenes investigadores 2014.

\section{References}

1. Liu, F.J. "He's fractional derivative for heat conduction in a fractal medium arising in silkworm cocoon hierarchy", Thermal Science, 19, pp. 1155-1159 (2015).

2. Oldham, K.B. and Spanier, J. "The fractional calculus", Academic Press, New York (1974).

3. Yao, J.J., Kumar, A., and Kumar, S. "A fractional model to describe the Brownian motion of particles and its analytical solution", Advances in Mechanical Engineering, 7(12), pp. 1-11 (2015).

4. Gómez-Aguilar, J.F., Razo-Hernández, R., and Granados -Lieberman, D. "A physical interpretation of fractional calculus in observables terms: analysis of the fractional time constant and the transitory response", Rev. Mex. Fís. 60, pp. 32-38 (2014).

5. Bia, P., Caratelli, D., Mescia, L., Cicchetti, R., Maione, G., and Prudenzano, F. "A novel FDTD formulation based on fractional derivatives for dispersive Havriliak-Negami media", Signal Processing, 107, pp. 312-318 (2015).

6. Mescia, L., Bia, P., and Caratelli, D. "Fractional derivative based FDTD modeling of transient wave propagation in Havriliak-Negami media", IEEE Transactions on Microwave Theory and Techniques, 62(9), pp. 1920-1929 (2014).

7. Ghaziani, R.K., Alidousti, J., and Eshkaftaki, A.B. "Stability and dynamics of a fractional order LeslieGower prey-predator model", Applied Mathematical Modelling, 40(3), pp. 2075-2086 (2016).

8. Caratelli, D., Mescia, L., Bia, P., and Stukach, O.V. "Fractional-calculus-based FDTD algorithm for ultrawideband electromagnetic characterization of arbitrary dispersive dielectric materials", IEEE Transactions on Antennas and Propagation, 64(8), pp. 35333544 (2016).

9. Kumar, S. "A new analytical modelling for fractional telegraph equation via Laplace transform", Applied Mathematical Modelling, 38(13), pp. 3154-3163 (2014).

10. Zingales, M. "Fractional-order theory of heat transport in rigid bodies", Communications in Nonlinear Science and Numerical Simulation, 19(11), pp. 39383953 (2014).

11. Mainardi, F., Luchko, Y., and Pagnini, G. "The fundamental solution of the space-time fractional diffusion equation", arXiv Preprint cond-mat/0702419 (2007).
12. Qi, H.T. and Jiang, X.Y. "Solutions of the spacetime fractional Cattaneo diffusion equation", Physica A. 390, pp. 1876-1883 (2011).

13. Liu, L., Zheng, L., Liu, F., and Zhang, X. "Heat conduction with fractional Cattaneo-Christov upperconvective derivative flux model", International Journal of Thermal Sciences, 112, pp. 421-426 (2017).

14. Ezzat, M.A., El-Bary, A.A., and Fayik, M.A. "Fractional Fourier law with three-phase lag of thermoelasticity", Mechanics of Advanced Materials and Structures, 20(8), pp. 593-602 (2013).

15. Zhao, J., Zheng, L., Zhang, X., and Liu, F. "Convection heat and mass transfer of fractional MHD Maxwell fluid in a porous medium with Soret and Dufour effects". International Journal of Heat and Mass Transfer, 103, pp. 203-210 (2016).

16. Narayan, O. and Ramaswamy, S. "Anomalous heat conduction in one-dimensional momentum-conserving systems", Physical review letters, 89(20), p. 200601 (2002).

17. Zheng, L., Liu, Y., and Zhang, X. "Slip effects on MHD flow of a generalized Oldroyd-B fluid with fractional derivative", Nonlinear Analysis: Real World Applications, 13(2), pp. 513-523 (2012).

18. Xiaojun, Y. and Baleanu, D. "Fractal heat conduction problem solved by local fractional variation iteration method", Thermal Science, 17(2), pp. 625-628 (2013).

19. Povstenko, Y.Z. "Fractional radial diffusion in a cylinder", Journal of Molecular Liquids, 137(1), pp. 46-50 (2008).

20. Abouelregal, A.E. "Fractional heat conduction equation for an infinitely generalized, thermoelastic, long solid cylinder", International Journal for Computational Methods in Engineering Science and Mechanics, 17(5-6), pp. 374-381 (2016).

21. Jiang, $\mathrm{X}$. and $\mathrm{Xu}, \mathrm{M}$. "The time fractional heat conduction equation in the general orthogonal curvilinear coordinate and the cylindrical coordinate systems", Physica A: Statistical Mechanics and its Applications, 389(17), pp. 3368-3374 (2010).

22. Atangana, A. "Derivative with two fractional orders: A new avenue of investigation toward revolution in fractional calculus", The European Physical Journal Plus, 131(10), pp. 1-13 (2016)

23. Liu, Y., Fang, Z., Li, H., and He, S. "A mixed finite element method for a time-fractional fourth-order partial differential equation", Applied Mathematics and Computation, 243, pp. 703-717 (2014).

24. Atangana, A. and Baleanu, D. "New fractional derivatives with nonlocal and non-singular kernel: Theory and application to heat transfer model", Therm Sci. 20(2), pp. 763-769 (2016).

25. Gómez-Aguilar, J.F., Rosales-García, J.J., BernalAlvarado, J.J., Córdova-Fraga, T., and GuzmánCabrera, R. "Fractional mechanical oscillators". Rev. Mex. Fis, 58(4), pp. 348-352 (2012). 
26. Mainardi, F. "An historical perspective on fractional calculus in linear viscoelasticity", Fractional Calculus and Applied Analysis, 15(4), pp. 712-717 (2012).

27. Povstenko, Y. "Fractional heat conduction and related theories of thermoelasticity", In Fractional Thermoelasticity, Springer International Publishing, pp. 13-33 (2015).

\section{Biographies}

José Francisco Gómez Aguilar received the BS and M.Eng. degrees in Electrical Engineering from Guanajuato University, Guanajuato, Mexico, in 2005 and 2007, respectively, and the PhD degree in Physics from División de Ciencias e Ingenierías, Guanajuato University, in 2012. He is currently a Full Research Professor with the Electronics Engineering Department commissioned for the CONACyT in the Centro Nacional de Investigación y Desarrollo Tecnológico, Tecnológico Nacional de México, Cuernavaca, Mexico. His current research interests include methods and applications of partial and ordinary differential equations, fractional differential equations, perturbations methods, image and signal processing, control, and power quality analysis.

Abdon Atangana obtained his BS degree in pure mathematics, his Honors degree and MS degree in
Applied Mathematics with distinction from University of Free State, South Africa. He also obtained his $\mathrm{PhD}$ degree in applied mathematics at the Institute for Groundwater Studies, University of the Free State, Bloemfontein, South Africa. He is serving as editor of some international journals, and also reviewer of more than 100 international accredited journals. He has been the Lead and Guest Editor of some special issues, and has presented and participated in more than 15 international conferences. His research interests are methods and applications of partial and ordinary differential equations, fractional differential equations, perturbations methods, asymptotic methods, iterative methods, and groundwater modeling. He is the author of the book "Derivative with new parameter: theory, methods and applications", published by Academic Press Elsevier.

Ricardo Escobar-Jiménez has received MS degree in Applied Science from the Centro de Investigación en Ingeniería y Ciencias Aplicadas and the PhD degree in science in electronic engineering from the Centro Nacional de Investigación y Desarrollo Tecnológico (CENIDET), Cuernavaca, Mexico, 2012. Actually, he is a Professor at CENIDET. His research interests are in nonlinear and linear modeling, fractional differential equations, control, fault diagnosis and their application in real systems. 